\title{
Comprendre la victoire de Jair Bolsonaro. Une revanche aveugle des élites contre le « lulisme»?
}

João Whitaker

\section{CpenEdition}

\section{Journals}

Édition électronique

URL : http://journals.openedition.org/ideas/5559

DOI : 10.4000/ideas.5559

ISSN : 1950-5701

Éditeur

Institut des Amériques

Référence électronique

João Whitaker, «Comprendre la victoire de Jair Bolsonaro. Une revanche aveugle des élites contre le « lulisme»? », IdeAs [En ligne], 13 | 2019, mis en ligne le 01 mars 2019, consulté le 24 avril 2019. URL http://journals.openedition.org/ideas/5559; DOI : 10.4000/ideas.5559

Ce document a été généré automatiquement le 24 avril 2019

\section{cc)}

IdeAs - Idées d'Amériques est mis à disposition selon les termes de la licence Creative Commons Attribution - Pas d'Utilisation Commerciale - Pas de Modification 4.0 International. 


\title{
Comprendre la victoire de Jair Bolsonaro. Une revanche aveugle des élites contre le « lulisme »?
}

\author{
João Whitaker
}

1 Ce serait une erreur de croire que la victoire de Jair Bolsonaro à la présidence du Brésil est le simple résultat de la vague conservatrice qui mène l'extrême droite au pouvoir de par le monde, à commencer par les États-Unis. Il est clair que la montée en puissance de ce mouvement conservateur a aidé, surtout au niveau des tactiques politiques et électorales mises en œuvre, notamment avec la présence au Brésil de Steve Bannon, la fraude des faux-messages de Whatsapp ( 350 millions de messages payés illégalement et envoyés avec des mensonges à des comptes ciblés) et, surtout, l'adoption du modèle de la post-vérité, où l'on peut dire, sans aucune inquiétude quant aux preuves ou aux références, n'importe quoi sur n'importe quel sujet.

2 Cependant, ces aspects sont périphériques pour comprendre la victoire de Jair Bolsonaro, qui repose plutôt sur des phénomènes sociaux et politiques endogènes. Il faut comprendre la structure sociale particulière de ce pays, et remonter un peu avant l'élection de Lula en 2002, pour appréhender toute la complexité du scénario actuel. La victoire de Lula avait acté la débâcle du modèle néolibéral porté par les élites brésiliennes depuis le tournant des années 1990. Le Parti des travailleurs (PT), porteur de promesses de changements sociaux structurels, emmenait au pouvoir pour la première fois, avec Lula, un représentant populaire légitime, et non pas un membre de l'élite patrimoniale traditionnelle. La bourgeoisie brésilienne n'est pas une bourgeoisie industrielle, au sens marxiste du terme, mais plutôt un groupe minoritaire de propriétaires, qui prend ses racines dans l'élite coloniale foncière et sa logique esclavagiste. Elle a ensuite évolué, devenant plus diversifiée et complexe, et regroupe aujourd'hui des grands propriétaires, des industriels, des banquiers et autres entrepreneurs médiatiques. Cette élite se maintient au pouvoir traditionnellement en instrumentalisant l'État selon ses intérêts 
particuliers. Au Brésil, selon les études récentes de Thomas Piketty, les $1 \%$ les plus riches détiennent près de $28 \%$ des richesses (le taux le plus élevé au monde). Quant à la concentration des terres dans les mains de quelques grands propriétaires, dont bon nombre occupent également des positions politiques de haut niveau, elle est l'une des plus accentuées au monde: les propriétés de plus de 200 hectares, qui représentent à peine $0,8 \%$ de l'ensemble, occupent $42,5 \%$ des terres cultivées (Dabène 0 ., Louault F., $2018: 70$ ).

3 Lula n'a pas vraiment changé cette structure sociale séculaire, mais a plutôt adopté une stratégie de "négociation » avec l'élite. Il a tout de même chamboulé l'ordre régnant et s'est attaqué, au moyen d'une série de politiques publiques, à une certaine "culture des privilèges » des classes moyennes et haute (politiques de redistribution, investissements dans les régions pauvres du Nord et du Nordeste, ouverture de l'accès aux universités, reconnaissance et l'espace politique donné aux mouvements sociaux en général, et plus particulièrement féministes, homosexuels, Noirs et Indiens, etc.). Dans le même temps, le succès économique incontestable des gouvernements Lula (2003-2010), quand le pays a retrouvé un niveau de croissance inédit depuis les années 1970, a permis une véritable révolution consommatrice, permettant à des millions de familles de classe moyennebasse de s'insérer dans les circuits de consommation, avec tout ce que cela implique. Ce cycle vertueux a permis au PT d'entretenir une dynamique électorale favorable. Cependant, et à l'inverse de la stratégie développée par exemple par Hugo Chávez au Venezuela, cette hausse du pouvoir d'achat n'a pas été accompagnée par une formation politique permettant à la population de développer une plus grande conscience politique.

4 En 2010, fort de son incontestable popularité, Lula parvient à faire reconnaitre Dilma Rousseff comme sa légitime successeure. Mais à peine entrée en fonctions, Rousseff se montre rapidement peu flexible aux négociations avec sa base parlementaire. En plus des difficultés politiques, Rousseff doit faire face à une conjoncture économique difficile. Elle peine à maintenir une politique de régulation face au pouvoir des banques, qui au Brésil pratiquent toujours les taux d'intérêts bancaires les plus élevés au monde. Elle ordonne aux deux banques publiques de pratiquer sur le marché des taux plus bas, déclarant la guerre à ce secteur puissant. Sa rigidité politique, même envers les mouvements sociaux qui la soutiennent, n'aide pas à sa popularité. Car le projet « développementiste » du PT, qui vise la croissance économique à tout prix, est séduit par la recette des grands projets urbains portés par l'organisation de grands évènements sportifs (Coupe du Monde de 2014 et Jeux Olympiques de 2016). Des projets qui, inévitablement, privilégient les grands chantiers d'infrastructure (transports, stades, etc.), avec un impact souvent néfaste sur les quartiers pauvres. Des mouvements d'opposition se structurent à gauche du PT et gagnent un certain poids. Malgré cela, Rousseff parvient à se faire réélire de justesse en 2014, dans un scénario déjà d'extrême polarisation.

5 Cette polarisation s'est construite autour de phénomènes croisés. En juin 2013, le pays est marqué par des manifestations souvent comparées au Printemps arabe. D'un profil jeune, apartisan, les manifestants ciblent d'abord les autorités politiques des grandes villes, responsables des politiques de transport, en raison d'une hausse des prix des billets de bus. Les élites, portées par les grandes entreprises et groupes de communication, voient là une opportunité pour créer un autre mouvement, celui-ci très générique, utilisant des slogans patriotiques et sans revendication précise, tourné directement contre la présidente Rousseff. Un ras-le-bol insufflé par la presse trouve rapidement écho parmi les 
classes moyennes. Des mouvements de rue, portés par des manifestants d'un genre nouveau qui portent le maillot jaune de la sélection nationale de football, gagnent en intensité. Dans la même séquence de 2013, des manifestants défendant le retour des militaires et vantant les «bienfaits » de la dictature, viennent eux aussi occuper l'espace public. Rousseff conserve néanmoins un appui populaire solide qui s'incarne dans des contre-manifestations massives (même si celles-ci ont été largement passées sous silence par les médias brésiliens). Cela explique en partie le fait que, malgré la polarisation, Rousseff remporte de justesse les élections de 2014. Les élites traditionnelles, qui avaient monopolisé le pouvoir jusqu'à l'élection de Lula en 2002, n'acceptent pas cette quatrième défaite consécutive face au PT. Le Parti de la social-démocratie brésilienne (PSDB), principal parti d'opposition, et certains secteurs du Parti du mouvement démocratique brésilien (PMDB) - qui occupe pourtant depuis 2011 la vice-présidence de la République aux côtés du PT - refusent le résultat des élections et enclenchent, dès le lendemain du second tour, une stratégie visant à fragiliser plus encore la présidente réélue. Profitant de la polarisation existante, ces groupes politiques lancent une campagne visant à détruire simultanément les images de Lula, de Rousseff et du PT, les présentant comme les « pires corrompus de l'histoire du pays ». L'affaire Petrobrás, qui éclate début 2015, leur donne du grain à moudre... Pourtant, le PT demeure l'un des partis politiques les moins impliqués dans des affaires de corruption durant ses expériences gouvernementales (dans les villes, les États et le gouvernement fédéral). Lula, puis Rousseff, se sont d'ailleurs employés à renforcer les institutions luttant contre la corruption: augmentation des moyens d'enquête de la police fédérale, indépendance de la justice, etc. Une donnée illustre cela: plus de deux mille actions anti-corruption ont été menées par la Police Fédérale sous les gouvernements du PT, contre une quarantaine lors des huit années du gouvernement Cardoso.

6 À court terme, l'objectif premier était de destituer, coûte que coûte, la présidente réélue. Après avoir monté une accusation superflue autour de technicités fiscales, et avec la complicité du président de la Chambre fédérale, les anti-pétistes parviennent à leurs fins en 2016. Le vice-président, Michel Temer, usurpe ainsi le pouvoir et fait entrer ses opposants de la veille au gouvernement. En un demi mandat, et sans légitimité électorale, ce gouvernement prend le contre-pied des politiques mises en place par Lula et Dilma: adoption d'un nouveau code du travail fragilisant les droits acquis des travailleurs, libéralisation des pesticides pour l'agrobusiness, arrêt de tous les investissements publics de caractère social pour les vingt années à venir, remise en cause des démarcations des territoires indigènes, etc. En parallèle, l'action incessante des médias pour nourrir «l'anti-pétisme» se montre efficace: le PT sort exsangue des élections municipales d'octobre 2016; il perd près des deux tiers de son électorat et des municipalités qu'il contrôlait. Un an et demi plus tard, Lula est emprisonné et mis hors du jeu électoral, malgré un manque de preuves tangibles. Rien ne semble plus empêcher les élites traditionnelles de confirmer leur reprise en main du pouvoir lors des élections générales d'octobre 2018. Mais la stratégie ne fonctionne pas exactement comme prévu. La société brésilienne ressent le coup porté aux institutions démocratiques. La polarisation se renforce. Si d'un côté, une masse impressionnante se regroupe contre ce qui est ressenti comme une double injustice (la destitution de Rousseff et l'emprisonnement de Lula), de l'autre côté se développe plutôt un ras-le-bol de la politique en général, ce qui inclut aussi les élites traditionnelles du PSDB et du PMDB. Alors qu'ils s'attendaient à cueillir les fruits de leur stratégie, ces deux partis se voient balayés dès le premier tour de l'élection présidentielle de 2018, recevant un pourcentage insignifiant de votes. Le PT reste la 
principale force politique de gauche, élisant le plus grand nombre de gouverneurs (cinq États) et de députés. Son candidat, Fernando Haddad, ex-maire de São Paulo et remplaçant officiel de Lula, obtient plus de 47 millions de votes au second tour. Mais contre les prévisions des stratèges du coup de 2016, c'est un outsider présenté comme d'extrême droite, l'ancien militaire Jair Bolsonaro, qui souffle la place et remporte l'élection.

7 Bolsonaro n'a pas d'idéologie à proprement parler. C'est un aventurier politique, un opportuniste, qui a siégé pendant vingt-huit ans en tant que député fédéral inexpressif. Durant ces trois décennies, son activité législative a été dérisoire (Louault F., 2018). Se prévalant des techniques de fausses nouvelles (fake news) dirigées par les spécialistes de Trump, le groupe de Bolsonaro a su jouer sur l'ignorance de certains groupes d'électeurs et sur la crédulité des masses évangélistes, dont les églises ont promptement adhéré à sa candidature. Des millions de messages ciblés, évoquant des affirmations fantaisistes (que Haddad allait distribuer aux bébés des biberons avec des tétines en forme de pénis, par exemple), ont fait des ravages parmi ces populations. Quant à la classe moyenne consommatrice, fatiguée par la corruption et le sentiment d'insécurité, il lui a été servi un discours sur mesure. Au sein de ces classes moyennes et supérieures, nombre d'hommes, blancs, d'âge moyen, se sentaient également menacés dans leurs privilèges par les politiques mises en œuvre sous les gouvernements du PT. Ces électeurs ne se sentaient plus à l'aise pour étaler leur misogynie, leur racisme, leur homophobie, leurs préjugés envers les plus pauvres. Ils en avaient assez des manifestations féministes - un des fils du président a récemment publié sur les réseaux sociaux l'hashtag «le féminisme est une maladie » (Sardinha E., 2018). Le politiquement correct est abominé, les armes sont adorées. Bolsonaro cultive depuis des années un discours machiste qui les fait exulter. Il incite à faire «fusiller les militants du PT » (Ribeiro J., 2018), explique que « la dictature aurait dû tuer beaucoup plus » et que l'erreur des tortionnaires du régime militaire a été de «torturer sans tuer» (Courrier international, 2018), etc. Dans son premier discours officiel, il annonce qu'il va « abolir le politiquement correct » (Planalto - Presidência da República, 2019). Toutes ces manifestations ne sont pas le portrait d'une pensée spécifique, mais la manière de faire de la politique d'un groupe de petits entrepreneurs politiques.

Car Jair Bolsonaro a monté avec grand soin un clan familial-politique dans son fief, la ville de Rio de Janeiro. De ses trois fils, l'un est conseiller municipal, l'autre est député de l'État de Rio, le troisième est député fédéral. Ensemble, ils constituent une petite entreprise familiale spécialisée dans la survie politique et l'enrichissement par des moyens plus ou moins clairs. Pendant la campagne, des informations au sujet d'importantes sommes d'argent (environ 1,5 million d'euros) transférées de manière suspecte par l'un des employés de la famille - un ex-policier devenu chauffeur du clan - ont ainsi été dévoilées (Gatinois C., 2018). Bolsonaro en a lui-même été avisé, mais l'affaire a été vite étouffée pour ne pas fragiliser sa candidature. Une fois le gouvernement en place, cependant, le scandale ne pouvait plus être contenu et a perturbé le début de mandat du nouveau président. D'autres affaires douteuses voient le jour, comme celle mettant à nu des relations aussi obscures que dangereuses entre le sénateur Flavio Bolsonaro (fils aîné du président) et les milices paramilitaires qui sévissent dans les favelas de Rio de Janeiro (Nassif L., 2019). Alors que le meurtrier de Marielle Franco est enfin emprisonné, le pays se surprend avec une photo du tueur aux cotés... du nouveau président, dont il est voisin (et ami ?) dans un condominium de luxe de Rio (Revista Forum, 2019). Quant au parti 
politique de Bolsonaro, le Parti social libéral (PSL), il est touché par un scandale de fausses candidatures pour détourner des fonds publics dans le cadre de la campagne électorale de 2018... Directement impliqué, le président du parti et secrétaire général du gouvernement, Gustavo Bebbiano, a été contraint de démissionner dès le mois de février 2019.

Complètement désorienté par ces affaires - mais aussi par l'inexpérience et l'incompétence de ses équipes gouvernementales - Bolsonaro n'a aucune ligne politique spécifique. Son gouvernement se compose de groupes contradictoires : militaires ultranationalistes, Chicago boys ultra-libéraux, pasteurs évangéliques, etc. En même temps, la nomination des autres ministres renforce le caractère hétéroclite et inédit du gouvernement Bolsonaro: un fonctionnaire méconnu au ministère des Affaires Étrangères, qui soutient que le réchauffement climatique est une conspiration globale menée par les Chinois, un ministre de l'Éducation qui défend l'installation d'écoles militaires comme gage de discipline et de sérieux, une ministre des Droits Humains qui ment sur ses diplômes (des diplômes "bibliques", précisera ensuite cette pasteure évangélique), qui déclare avoir vu Jésus sur un arbre et que le pays est sauvé car maintenant « les garçons vont vêtir du bleu et les filles, du rose » (O Globo, 2019), etc. Dans la pratique, des décisions ont déjà été prises, comme le décret unilatéral signé par le président, que beaucoup considèrent inconstitutionnel, de libéraliser la vente d'armes aux particuliers. Au milieu de tout cela, le nouveau gouvernement semble s'effondrer dans des disputes internes dès les cent premiers jours au pouvoir.

\section{BIBLIOGRAPHIE}

Courrier international, « Dix citations qui en disent long sur Jair Bolsonaro », Courrier international, 31 octobre 2018. URL : https://www.courrierinternational.com/article/dixcitations-qui-en-disent-long-sur-jair-bolsonaro

Dabène, Olivier et Louault, Frédéric, Atlas du Brésil. Promesses et défis d'une puissance émergente, Paris, Autrement, $2^{\mathrm{e}}$ édition, 2018.

Gatinois, Claire, «Brésil : Jair Bolsonaro, pas encore en fonction, déjà rattrapé par les affaires ", Le Monde, 19 décembre 2018. URL : https://www.lemonde.fr/international/article/2018/12/19/ bresil-jair-bolsonaro-pas-encore-en-fonction-deja-rattrape-par-les-affaires_5399562_3210.html

Louault, Frédéric, « Portrait de Jair Bolsonaro, président de la République Fédérative du Brésil », Blog Institut Montaigne, 21 décembre 2018. URL : https://www.institutmontaigne.org/blog/ portrait-de-jair-bolsonaro-president-de-la-republique-federative-du-bresil

Nassif, Luis, « Xadrez do fim do governo Bolsonaro », Jornal GGN, 20 janvier 2019. URL : https:// jornalggn.com.br/eleicoes/xadrez-do-fim-do-governo-bolsonaro-por-luis-nassif/

O Globo, « Relembre as polêmicas envolvendo Damares Alves em um mês de gestão », O Globo, 31 janvier 2019. URL : https://oglobo.globo.com/sociedade/relembre-as-polemicas-envolvendodamares-alves-em-um-mes-de-gestao-23416699 
Planalto - Presidência da República, « Discurso do Presidente da República, Jair Bolsonaro, durante Cerimônia de Posse no Congresso Nacional », $1^{\text {er }}$ janvier 2019 ; URL : http:// www2.planalto.gov.br/acompanhe-o-planalto/discursos/2019/discurso-do-presidente-darepublica-jair-bolsonaro-durante-cerimonia-de-posse-no-congresso-nacional

Revista Forum, « Preso por morte de Marielle publicou foto com Bolsonaro no Facebook ; perfil desaparece da rede », Revista Forum, 12 mars 2019. URL : https://www.revistaforum.com.br/ preso-por-morte-de-marielle-publicou-foto-com-bolsonaro-no-facebook-perfil-desaparece-darede/

Ribeiro, Janaína, " "Vamos fuzilar a petralhada", diz Bolsonaro em campanha no Acre », Exame, 04 septembre 2018. URL : https://exame.abril.com.br/brasil/vamos-fuzilar-a-petralhada-dizbolsonaro-em-campanha-no-acre/

Sardinha, Edson, « Palavrões, ameaças e o conflito sentimental que levaram Eduardo Bolsonaro a ser denunciado ", Congresso em foco, 14 avril 2018. URL : https://congressoemfoco.uol.com.br/ especial/noticias/palavroes-ameacas-e-o-conflito-sentimental-que-levou-eduardo-bolsonaro-aser-denunciado/

\section{AUTEUR}

\section{JOÃO WHITAKER}

Professeur-chercheur à l'Université de São Paulo, faculté d'architecture et urbanisme (FAU), où il est président de l'école doctorale en architecture et urbanisme. Il est l'auteur, entre autres, du livre Le mythe de la ville globale, et a été Secrétaire municipal au Logement de la ville de São Paulo, sous la gestion de Fernando Haddad. Il fut professeur invité à l'IHEAL en 2011. 